\title{
Asymptotically Fast Computation of Hermite Normal Forms of Integer Matrices
}

\author{
Arne Storjohann and George Labahn \\ Department of Computer Science \\ University of Waterloo, Ontario, Canada \\ \{astorjoh,glabahn\}@daisy.uwaterloo.ca
}

\begin{abstract}
This paper presents a new algorithm for computing the Hermite normal form $H$ of an $A \in \mathbf{Z}^{n \times m}$ of rank $m$ together with a unimodular pre-multiplier matrix $U$ such that $U A=H$. Our algorithm requires $O^{\sim}\left(m^{\theta-1} n \mathrm{M}(m \log \|A\|)\right)$ bit operations to produce both $H$ and $U$. Here, $\|A\|=$ $\max _{\imath \jmath}\left|A_{i j}\right|, M(t)$ bit operations are sufficient to multiply two $[t]$-bit integers, and $\theta$ is the exponent for matrix multiplication over rings: two $m \times m$ matrices over a ring $\mathrm{R}$ can be multiplied in $O\left(m^{\theta}\right)$ ring operations from $\mathrm{R}$. The previously fastest algorithm of Hafner \& McCurley requires $O^{-}\left(m^{2} n \mathrm{M}(m \log \|A\|)\right)$ bit operations to produce $H$, but does not produce a unimodular matrix $U$ which satisfies $U A=H$. Previous methods require on the order of $O^{\sim}\left(n^{3} M(m \log \| A||)\right)$ bit operations to produce a $U-$ our algorithm improves on this significantly in both a theoretical and practical sense.
\end{abstract}

\section{Introduction}

A fundamental notion for matrices over rings is left equivalence. Two $n \times m$ matrices $A$ and $B$ over a principal ideal ring $\mathrm{R}$ are said to be left equivalent if there exists an $n \times n$ unimodular matrix $U$ that satisfies $U A=B$. (A unimodular matrix has determinant a unit in $\mathrm{R}$ and hence is invertible.) Any integer matrix $A$ with full column rank can be transformed to an upper triangular matrix $T$ using only elementary row operations. The triangularization $T$ can be made unique by enforcing that diagonal entries be positive and off-diagonal entries be non-negative and reduced in magnitude modulo the diagonal entry in each column. In particular, any $A \in \mathbf{Z}^{n \times m}$ with rank $m$ is left equivalent to a unique upper triangular matrix $H$. That is, there exists a unimodular matrix $U$ (i.e. $\operatorname{det}(U) \pm 1$ ) such that

Permission to make digital/hard copies of all or part of this material for personal or classroom use is granted without fee provided that the copies are not made or distributed for profit or commercial advantage, the copyright notice, the title of the publication and its date appear, and notice is given that copyright is by permission of the ACM, Inc. To copy otherwise, to republish, to post on servers or to redistribute to lists, requires specific permission and/or fee. ISSAC'96, Zurich, Switzerland; (C1996 ACM 0-89791-796-0/96/07. . \$3.50

$$
U A=H=\left[\begin{array}{ccccc}
h_{1} & \bar{h}_{12} & \bar{h}_{13} & & \bar{h}_{1 m} \\
& h_{2} & \bar{h}_{23} & \cdots & \bar{h}_{2 m} \\
& & h_{3} & & \bar{h}_{3 m} \\
& & & \ddots & \vdots \\
& & & & h_{m} \\
& & & &
\end{array}\right]
$$

where $h_{j}$ is positive for $1 \leq j \leq m$ and $\bar{h}_{i j}$ satisfies $0 \leq$ $\bar{h}_{i j}<h_{j}$ for $1 \leq i, j \leq m$. The reduced triangularization $\bar{H}$ is called the Hermite normal form of $A$ and the unimodular $U$ is called a pre-multiplier matrix. The Hermite normal form was first proven to exist by Hermite [5, 1851] for the case of a square nonsingular input matrix. The Hermite normal form is in fact a canonical form for left equivalence over $\mathbf{Z}$ - it always exists and is unique (see, for example, Newman $[10,1972])$.

Hafner \& McCurley $[4,1991]$ have given an algorith$\mathrm{m}$ that requires $O^{\sim}\left(m^{2} n \mathrm{M}(m \log \|A\|)\right)^{1}$ bit operations to compute the Hermite normal form of $A$. They have also shown how to apply fast matrix multiplication techniques to the problem of triangularizing matrices over principal ideal rings. They show how to apply their result to the case $\mathrm{R}=\mathbf{Z}$ to get an asymptotically fast algorithm for obtaining a unimodular triangularization of an integral input matrix. This results in an algorithm that requires $O^{\sim}\left(m^{\theta-1} n \mathrm{M}(m \log \|A\|)\right)$ bit operations to produces an upper triangular $T$ left equivalent to $A$. Here $\theta$ denotes the number such that two $m \times m$ matrices over a ring $\mathrm{R}$ can be multiplied in $O\left(m^{\theta}\right)$ ring operations from R. Using standard multiplication $\theta=3$, while the best known algorithm of Coppersmith \& Winograd $[2,1990]$ allows $\theta=2.38$. However, Hafner \& McCurley were unable to obtain an algorithm to compute the complete Hermite normal form with the improved complexity.

For some applications the complete Hermite normal form of an input matrix is not required; a general triangularization such as produced by Hafner \& McCurleys algorithm may be sufficient. The Hermite normal form, however, has some important advantages over a general triangularization. First, the Hermite normal form is a canonical form for left equivalence. To determine whether a second matrix $B$ is

\footnotetext{
${ }^{1}$ To summarize results we use "soft-Oh" notation: for any $f, g$ : $\Re^{s} \mapsto \Re, f=O^{\sim}(g)$ if and only if $f=O\left(g \cdot \log ^{c} g\right)$ for some constant $c>0$.
} 
left equivalent to $A$, it is sufficient to compare the Hermite normal forms of $A$ and $B$. This check for left equivalence is not possible using a general (non unique) triangularization. Secondly, the space required to write down the Hermite normal form $H$ of $A$ will be small compared to that of general triangularization $T$. Consider the case of a square nonsingular input matrix $A \in \mathbf{Z}^{n \times n}$. The total size of $H$ (the sum of the bit lengths of the individual entries of $H$ ) will be on the order of $O^{-}\left(n^{2} \log \|A\|\right)$ bits, or about the same space as required to write down the input matrix. The triangularization $T$ returned by Hafner \& McCurleys algorithm will have entries bounded in length by $\left.O^{\sim}(n \log \|A\|)\right)$ bits. This leads to a total size bound for $T$ of $O^{\sim}\left(n^{3} \log \|A\|\right)$ bits, or about a factor $n$ larger than $H$. Since $A$ is nonsingular, there will be a unique unimodular matrix $U$ which satisfies $U A=H$ and $A=H U^{-1}$. Similarly, there exists a unique unimodular $P$ with $P A=T$ and $A=T P^{-1}$. Entries in $U$ and $U^{-1}$ will be bounded in length by $O^{-}(n \log \|A\|)$ bits and by $O^{\sim}(\log \|A\|+\log n)$ bits respectively. Entries of $P$ and $P^{-1}$, on the other hand, are bounded in length by $O^{-}(n \log \|A\|)$ bits and $O^{\sim}\left(n^{2} \log \|A\|\right)$ bits respectively.

In this paper we show how to use a fast matrix multiplication decomposition for reducing off-diagonal entries of an upper triangular matrix $T$. Combining our result with Hafner \& McCurleys triangularization method gives an algorithm for computing the Hermite normal form $H$ of $A$ in $O^{\sim}\left(m^{\theta-1} n \mathrm{M}(m \log \|A\|)\right)$ bit operations.

In addition, we show how to recover a unimodular premultiplier matrix $U$ which satisfies $U A=H$. Computing a pre-multiplier is required in such applications as integer programming $[6,1969]$, solving linear diophantine equations $[8,1989]$ and computing matrix greatest common divisors $[12,1995]$. In the case where $A$ is square and nonsingular, $U$ is unique and can be recovered with no increase in asymptotic complexity by computing $U \leftarrow H A^{-1}$ using standard methods. However, for the rectangular case where $A$ is $n \times m$ with $n>m$, the pre-multiplier matrix $U$ is not unique. Our algorithm recovers both $H$ and an $n \times n$ unimodular $U$ in $O^{-}\left(m^{\theta-1} n \mathrm{M}(m \log \|A\|)\right)$ bit operations. The previously fastest algorithm for Hermite normal form, which works modulo the determinant of the input matrix to prevent expression swell, has initially been presented for the case of square nonsingular input matrices (see, for example, Domich, Kannan \& Trotter [3, 1987] or Iliopolous $[9,1989])$. Hafner \& McCurley [4, 1991] extend the mod determinant approach and give an algorithm that requires $O^{\sim}\left(m^{2} n \mathrm{M}(m \log \|A\|)\right)$ bit operations to produce $H$, but they don't show how to produce a suitable $U$ within this time. They suggest the following scheme for producing a $U$. Permute the rows of $A$ and augment with the $n-m$ identity matrix to get a new $n \times n$ matrix $\vec{A}$ which can be written in block form as

$$
\bar{A}=\left[\begin{array}{l|l}
A_{1} & \\
\hline A_{2} & I_{n-m}
\end{array}\right]
$$

where $A_{1}$ is nonsingular. Compute the Hermite normal form $\bar{H}$ of $\bar{A}$ at a cost of $O^{\sim}\left(n^{3} \mathrm{M}(m \log \|A\|)\right)$ bit operations, and set $U \leftarrow \bar{H} \bar{A}^{-1}$. The algorithm we give in this paper improves this worst case complexity bound by a factor of about $O\left(n^{2} / m^{\theta-1}\right)$ - a significant improvement for rectangular matrices even assuming standard matrix multiplication. Moreover, the matrix $U$ will produced by our algorithm will be "nice". By this we mean that the entries of $U$ will be bounded in length by $O^{\sim}(m \log \|A\|)$ bits and
$U$ will be sparse, with on the order of only $\mathrm{O}^{-}(\mathrm{nm})$ nonzero integer entries.

\section{Preliminaries and Previous Results}

Following Hafner \& McCurley in [4, 1991], we will express our complexity results in terms of a function $\mathrm{B}(t)$ that bounds the number of bit operations required to solve both the extended Euclidean problem with two $\lceil t\rceil$ bit integers and to apply the Chinese remainder algorithm with moduli consisting of all primes less than $t$. By Theorem 8.20 and 8.21 of Aho, Hopcroft \& Ullman $[1,1974]$ we can take $\mathrm{B}(t) \ll M(t) \log (t)$ where $\mathrm{M}(t)$ is a monotonic upper bound on the number of bit operations required to multiply two $\lceil t\rceil$ bit integers. The Schönhage \& Strassen $[11,1971]$ integer multiplication algorithm allows $M(t) \ll t \log t \log \log t$ hence

$$
\mathrm{B}(t) \ll t \log ^{2} t \log \log t
$$

In what follows, we write $\mathrm{MM}(n)=\mathrm{MM}_{\mathrm{R}}(n)$ to mean the number of ring operations required to multiply two $n \times n$ matrices over a ring $R$, where

$$
\operatorname{MM}(n) \ll n^{\theta} .
$$

It is well known that the product of the diagonal entries in the Hermite normal form of an $n \times m$ matrix $A$ with rank $m$ is given by the gcd of all $m \times m$ minors of $A-$ in what follows we refer to this quantity as $\operatorname{det}(\mathcal{L}(A))$. We end this section with a result of Hafner \& McCurley $[4,1991]$ that will be required in Sections 3 and 4 .

Lemma 1 (Hafner \& McCurley $([\mathbf{4}, \mathbf{1 9 9 1}])$ There exists a deterministıc algorithm that takes as input an $n \times m$ rank $m$ integral matrix $A$ and positive integer $d^{\prime}$ that is a multiple $\operatorname{det}(\mathcal{L}(A))$, and produces as output an upper triangular matrix $T$ that is left equivalent to $A$. Entries in $T$ will be bounded in magnitude by $d$ and the running time of the algorithm is $O\left(m n \mathrm{~B}(\log \|A\|)+m^{\theta-1} n \log (2 n / m) \mathrm{B}(\log d)\right)$ bit operations.

Hafner \& McCurley also show also how to obtain a suitable multiple $d$ of $\operatorname{det}(\mathcal{L}(A))$ which satisfies $d \leq m^{m / 2}\|A\|^{m}$.

Theorem 2 (Hafner \& McCurley [4, 1991]) There exists a deterministic algorithm that takes as input an $n \times m$ rank $m$ integral matrix $A$, and produces as output an upper triangular matrix $T$ that is left equivalen$t$ to $A$. Entries in $T$ will be bounded in magnitude by $m^{m / 2}\|A\|^{m}$, and the running time of the algorithm is $O\left(m^{\theta-1} n \log (2 n / m) \mathrm{B}(m \log m\|A\|)\right)$ bit operations.

\section{Asymptotically Fast Hermite Normal Form}

In this section we give our asymptotically fast algorithm for computing the Hermite normal form of an $A \in \mathbf{Z}^{n \times m}$. First we apply the triangularization algorithm of Theorem 2 to transform $A$ to an upper triangular $T \in \mathbf{Z}^{m \times m}$ having diagonal entries the same as those in the Hermite normal form of $A$. Our approach is to consider $T$ as a matrix over $\mathbf{Z}_{d}$, where $d$ is a positive integer multiple of $\operatorname{det}(T)$. In particular, let $\vec{T}$ be the matrix obtained from $T$ by reducing each entry modulo $d$. In Subsection 3.1 we present an algorithm which computes a unit upper triangular $\bar{U} \in \mathbf{Z}_{d}^{n \times n}$ which satisfies $\bar{U} \bar{T}=H \bmod d$, where $H$ is in Hermite normal form over $\mathbf{Z}_{d}$, that is, has off-diagonal entries in each 
column reduced modulo the diagonal entry in each column. The Hermite normal form of $T$ is simply $H$, considered now over $\mathbf{Z}$ rather than $\mathbf{Z}_{d}$. In Subsection 3.2 we make this argument precise and give the complete Hermite normal form algorithm

\subsection{Hermite Normal Form of a Triangular Matrix}

In this subsection we work completely over the ring $\mathbf{Z}_{d}$. In particular, all matrices will be over $\mathbf{Z}_{d}$, and equations should be taken to hold modulo $d$. For brevity, we give our complexity results in terms of the number of operations from $\mathbf{Z}_{d}$ - a single operation from $\mathbf{Z}_{d}$ has cost $O(\mathrm{~B}(\log d))$ bit operations.

Let $R(n)=R_{d}(n)$ be a bound on the number of operations from $\mathbf{Z}_{d}$ required to compute, for an upper triangular $T \in \mathbf{Z}_{d}^{n \times n}$ with nonzero diagonal entries, a unit upper triangular pre-multiplier $U$ such that $U T=H$, with $H$ in Hermite normal form. Our result is the following.

Theorem $3 R(n) \ll n^{\theta}$.

We prove Theorem 3 by reducing to a special case for which we require some notation. For $n$ even, let $\mathcal{T}_{n}$ be the set of $n \times n$ nonsingular upper triangular matrices over $\mathbf{Z}_{d}$ that can be written in block form as

$$
\left[\begin{array}{l|l}
I & A \\
\hline & B
\end{array}\right]
$$

where $A$ and $B$ are $(n / 2) \times(n / 2), B$ is in Hermite normal form, and the empty block is used to denote the zero matrix. Let $R^{*}(n)=R_{d}^{*}(n)$ be the number of ring operations required to compute, for a $T \in \mathcal{T}_{n}$, candidates for $(n / 2) \times(n / 2)$ matrices $Q$ and $R$ over $\mathbf{Z}_{d}$ such that

$$
\left[\begin{array}{c|c}
I & -Q \\
\hline & I
\end{array}\right]\left[\begin{array}{c|c}
I & A \\
\hline & B
\end{array}\right]=\left[\begin{array}{l|l}
I & R \\
\hline & B
\end{array}\right]
$$

with the matrix on the right hand side in Hermite normal form.

Lemma 4 For $n$ a power of 2, we have

$$
R^{*}(n) \ll n^{\theta}
$$

Proof We show how to reduce the problem to four subproblems of half the size which can be combined using matrix multiplication. We claim that

$$
\begin{aligned}
R^{*}(n) & \leq 4 R^{*}(n / 2)+\mathrm{MM}(n) \\
& \leq 4 R^{*}(n / 2)+c n^{\theta}
\end{aligned}
$$

for some absolute constant $c$. The second line in this inequality follows from (1). To prove (2), we start with a $T \in \mathcal{T}_{n}$, which, using a block decomposition, we can write as

$$
T=\left[\begin{array}{l|l|l|l}
I & & A_{1} & A_{3} \\
\hline & I & A_{2} & A_{4} \\
\hline & & B_{1} & B_{2} \\
\hline & & & B_{3}
\end{array}\right]
$$

where all blocks are of size $(n / 4) \times(n / 4)$. At a cost of $2 R^{*}(n / 2)$, compute $(n / 4) \times(n / 4)$ matrices $Q_{1}, R_{1}, Q_{2}$ and $R_{2}$ such that

$$
\left[\begin{array}{c|c}
I & -Q_{1} \\
\hline & I
\end{array}\right]\left[\begin{array}{l|l}
I & A_{1} \\
\hline & B_{1}
\end{array}\right]=\left[\begin{array}{l|l}
I & R_{1} \\
\hline & B_{1}
\end{array}\right]
$$

and

$$
\left[\begin{array}{c|c}
I & -Q_{2} \\
\hline & I
\end{array}\right]\left[\begin{array}{l|l}
I & A_{2} \\
\hline & B_{1}
\end{array}\right]=\left[\begin{array}{l|l}
I & R_{2} \\
\hline & B_{1}
\end{array}\right]
$$

with the matrices on the right hand side in Hermite normal form. At a cost of $\operatorname{MM}(n)$, compute the matrix product

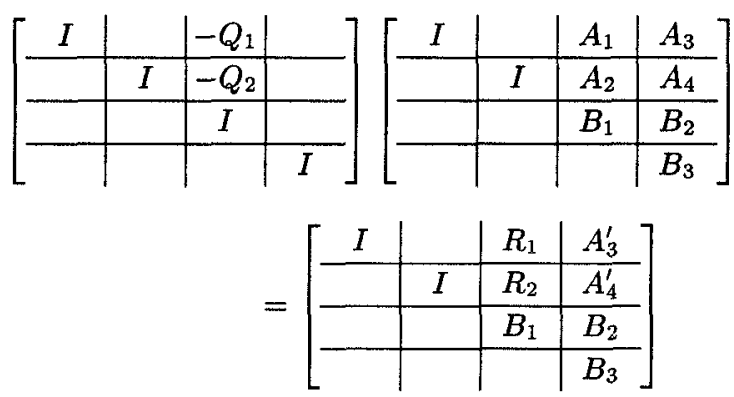

to get the transformed $(n / 4) \times(n / 4)$ blocks $A_{3}^{\prime}$ and $A_{4}^{\prime}$. The last stage is now similar to the first. At a cost of $2 R^{*}(n / 2)$, compute $(n / 4) \times(n / 4)$ matrices $Q_{3}, R_{3}, Q_{4}$ and $R_{4}$ such that

$$
\left[\begin{array}{c|c}
I & -Q_{3} \\
\hline & I
\end{array}\right]\left[\begin{array}{l|l}
I & A_{3}^{\prime} \\
\hline & B_{3}
\end{array}\right]=\left[\begin{array}{l|l}
I & R_{3} \\
\hline & B_{3}
\end{array}\right]
$$

and

$$
\left[\begin{array}{c|c}
I & -Q_{4} \\
\hline & I
\end{array}\right]\left[\begin{array}{c|c}
I & A_{4}^{\prime} \\
\hline & B_{3}
\end{array}\right]=\left[\begin{array}{l|l}
I & R_{4} \\
\hline & B_{3}
\end{array}\right]
$$

with the matrices on the right hand side in Hermite normal form. We now have

$\left[\begin{array}{c|c|c|c}I & & -Q_{1} & -Q_{3} \\ \hline & I & -Q_{2} & -Q_{4} \\ \hline & & I & \\ \hline & & & I\end{array}\right]\left[\begin{array}{c|c|c|c}I & & A_{1} & A_{3} \\ \hline & I & A_{2} & A_{4} \\ \hline & & B_{1} & B_{2} \\ \hline & & & B_{3}\end{array}\right]$

$=\left[\begin{array}{c|c|c|c}I & & R_{1} & R_{3} \\ \hline & I & R_{2} & R_{4} \\ \hline & & B_{1} & B_{2} \\ \hline & & & B_{3}\end{array}\right]$

with the premultiplier matrix on the left unit upper triangular and the matrix on the right hand side in Hermite normal form. This shows that

$$
R^{*}(n) \leq 4 R^{*}(n / 2)+\mathrm{MM}(n)
$$

which verifies (2). Iterate (2) to obtain

$$
\begin{aligned}
R^{*}(n) & \leq 4 R^{*}(n / 2)+c n^{\theta} \\
& =16 R^{*}(n / 4)+c n^{\theta}+4 c(n / 2)^{\theta} \\
& \vdots \\
& =4^{\left(\log _{2} n\right)-1} R^{*}(2)+c \sum_{i=0}^{\left(\log _{2} n\right)-2}\left(\frac{4}{2^{\theta}}\right)^{i} \\
& \ll n^{2} R^{*}(2)+n^{\theta} .
\end{aligned}
$$


The Lemma now follows since the cost of $R^{*}(2)$ is $O(1)$ operations from $\mathbf{Z}_{d}$.

We now return to the proof of Theorem 3 . By embedding an $n \times n$ upper triangular nonsingular input matrix $T$ into the block diagonal matrix $\operatorname{diag}\left(I_{p}, T\right)$, with $p$ a nonnegative integer bounded by $n$, we can assume without loss of generality that $n$ is a power of two. We claim that

$$
\begin{aligned}
R(n) & \leq 2 R(n / 2)+\mathrm{MM}(n)+\mathrm{MM}(n / 2)+R^{*}(n) \\
& \leq 2 R(n / 2)+c n^{\theta}
\end{aligned}
$$

for some absolute constant $c$. The second line of the inequality follows from (1) and Lemma 4 . To prove (3), we start with an $n \times n$ nonsingular upper triangular matrix $T$, which, using a block decomposition, we can write as

$$
T=\left[\begin{array}{l|l}
B_{1} & A_{2} \\
\hline & B_{2}
\end{array}\right] .
$$

At a cost of $2 R(n / 2)$, compute $(n / 2) \times(n / 2)$ matrices $U_{1}$, $H_{1}, U_{2}$ and $H_{2}$ such that $U_{1} B_{1}=H_{1}$ and $U_{2} B_{2}=H_{2}$ with $H_{1}$ and $H_{2}$ in Hermite normal form and $U_{1}$ and $U_{2}$ unit upper triangular. At a cost of $\operatorname{MM}(n)$, compute the matrix product

$$
\left[\begin{array}{l|l}
H_{1} & A_{2}^{\prime} \\
\hline & H_{2}
\end{array}\right]=\left[\begin{array}{l|l}
U_{1} & \\
\hline & U_{2}
\end{array}\right]\left[\begin{array}{l|l}
B_{1} & A_{2} \\
\hline & B_{2}
\end{array}\right] .
$$

At a cost of $R^{*}(n)$, compute $(n / 2) \times(n / 2)$ matrices $Q$ and $R_{2}$ such that

$$
\left[\begin{array}{c|c}
I & -Q \\
\hline & I
\end{array}\right]\left[\begin{array}{l|l}
I & A_{2}^{\prime} \\
\hline & \tilde{H}_{2}
\end{array}\right]=\left[\begin{array}{l|l}
I & R_{2} \\
\hline & H_{2}
\end{array}\right] .
$$

Finally, at a cost of $\mathrm{MM}(n / 2)$, compute the matrix $Q^{\prime}$ as $Q^{\prime} \leftarrow-Q U_{2}$. We now have

$$
\left[\begin{array}{l|l}
U_{1} & Q^{\prime} \\
\hline & U_{2}
\end{array}\right]\left[\begin{array}{l|l}
B_{1} & A_{2} \\
\hline & B_{2}
\end{array}\right]=\left[\begin{array}{l|l}
H_{1} & R_{2} \\
\hline & H_{2}
\end{array}\right]
$$

with the premultiplier matrix on the left unit upper triangular and the matrix on the right hand side in Hermite normal form. This shows that

$$
R(n) \leq 2 R(n / 2)+\mathrm{MM}(n)+\mathrm{MM}(n / 2)+R^{*}(n)
$$

which verifies $(3)$.

Iterate (3) to obtain

$$
\begin{aligned}
R(n) & \leq 2 R(n / 2)+c n^{\theta} \\
& =4 R(n / 4)+c n^{\theta}\left(1+2(1 / 2)^{\theta}\right) \\
& \vdots \\
& =2^{\left(\log _{2} n\right)-1} R(2)+c n^{\theta} \sum_{i=0}^{\left(\log _{2} n\right)-1}\left(2 / 2^{\theta}\right)^{\imath} \\
& \ll n^{2} R(2)+n^{\theta} .
\end{aligned}
$$

The result now follows since the cost of $R(2)$ is $O(1)$ operations from $\mathbf{Z}_{d}$.

\subsection{The Hermite Normal Form Algorithm}

Theorem 5 There exists a deterministic algorithm that takes as input an $n \times m$ rank $m$ integral matrix $A$, and produces as output the Hermite normal form of A. The running time of the algorithm is bounded by $O\left(m^{\theta-1} n \log (2 n / m) \mathrm{B}(m \log m\|A\|)\right)$ bit operations.
Proof Use the algorithm of Theorem 2 to produce an upper triangular $T$ left equivalent to $A$. As an intermediate step, the algorithm of Theorem 2 computes a positive integer multiple $d^{\prime}$ of $\operatorname{det}(\mathcal{L}(A))$ with $d^{\prime} \leq m^{m / 2}\|A\|^{m}$. Let $\bar{T}$ be the matrix obtained from $A$ by reducing entries modulo $d$, where $d=2 d^{\prime}$, and compute the Hermite normal form $H$ of $\bar{T}$ over $\mathbf{Z}_{d}$ using the algorithm of Theorem 3. So far, the running time is seen to be bounded by $O\left(m^{\theta-1} n \log (2 n / m) \mathrm{B}(m \log m\|A\|)\right)$ bit operations. The following lemma shows that the Hermite normal form of $T$, and hence $A$, is also $H$, considered now over $\mathbf{Z}$ rather than $\mathbf{Z}_{d}$.

Lemma 6 Let $\bar{U}, T$ and $H$ be $m \times m$ integral matrices with $\bar{U}$ unit upper triangular, $T$ upper triangular with positive diagonal entries and $H$ in Hermite normal form. If

$$
\bar{U} T=H \bmod 2 \operatorname{det}(T),
$$

then $H$ is the Hermite normal form of $T$.

Proof Since the Hermite normal form is a canonical form for left equivalence, we will be finished if we show their exists a $U \in \mathbf{Z}^{m \times m}$ which is unimodular and satisfies $U T=H$. It follows from (4) that the matrix $\bar{U} T-H$ has all entries divisible by $2 \operatorname{det}(T)$. Set $U \leftarrow \bar{U}-(1 /(2 \operatorname{det}(T))(\bar{U} T-$ $H)\left(2 T^{\text {adj }}\right)$. Then $U$ is integral and

$$
\begin{aligned}
U T & =\left(\bar{U}-(1 /(2 \operatorname{det}(T)))(\bar{U} T-H)\left(2 T^{\mathrm{adj}}\right)\right) T \\
& =\bar{U} T-(\bar{U} T-H)(1 / \operatorname{det}(T)) T^{\operatorname{adj}_{T}} \\
& =\bar{U} T-(\bar{U} T-H) \\
& =H .
\end{aligned}
$$

Since $\bar{U}$ is unit upper triangular and $2 \operatorname{det}(T)$ is strictly larger than each diagonal entry of $T$, the matrix $H=$ $U T \bmod 2 \operatorname{det}(T)$ will have the same diagonal entries as $T$ In particular, this means that $\operatorname{det}(H)=\operatorname{det}(T)$ and it follows from the identity $U T=H$ that $U$ is unimodular.

\section{Asymptotically Fast Pre-multiplier}

In this section we consider the problem of computing, for an $n \times m$ rank $m$ integral input matrix $A$, an $n \times n$ unimodular pre-multiplier matrix $U$ that satisfies $U A=H$. Our approach is based on an algorithm given by Hafner \& McCurley [4, 1991] for triangularizing matrices over rings. Combining one of their results, essentially the algorithm of Theorem 2, with the result of Section 3 leads directly to an algorithm that computes an $n \times n$ matrix $\hat{U}$ that satisfies

$$
\hat{U} A=H \bmod d
$$

for some positive integer multiple $d$ of $\operatorname{det}(\mathcal{L}(A))$. The cost of producing $\hat{U}$ will be bounded by $O^{\sim}\left(m^{\theta-1} n \mathrm{~B}(m \log m\|A\|)\right)$ bit operations. Note that this complexity result is almost linear in $n$ even though the output includes the $n \times n$ matrix $\hat{U}$. It turns out the the matrix $\hat{U}$ produced is sparse, with only $O(n m \log (2 n / m))$ nonzero entries. Unfortunately, equation (5) will not hold over $\mathbf{Z}$, nor will $\operatorname{det}(\hat{U})= \pm 1$.

The result of this section is a modification of Hafner \& McCurley's triangularization algorithm that produces an $n \times n$ integral matrix $U$ that satisfies both $U A=H$ and $\operatorname{det}(U)= \pm 1$. The algorithm also requires only $O^{\sim}\left(m^{\theta-1} n \mathrm{~B}(m \log m\|A\|)\right)$ bit operations. Moreover, $U$ will 
have entries bounded in length by $O(\log (2 n / m) m \log m|| A||)$ bits. The major portion of our work will go into ensuring that the size of intermediate integers occurring during the construction of $U$ do not become too large.

Our algorithm has two steps which we present separately in Subsection 4.1 and 4.2. In Subsection 4.1 we present a fast preconditioning step which ensures that certain $m \times m$ minors of the input matrix are nonsingular - we require this to properly bound the size of intermediate integers during the remainder of the algorithm. In Subsection 4.2 we present our algorithm for a special type of rectangular input matrix that can be decomposed using a block decomposition into $m \times m$ Hermite normal form matrices. Finally, we combine these results in Subsection 4.3 and present the general algorithm.

Before continuing, we present some simple results which will be required later on. The following result establishes some useful bounds on the magnitudes of entries occurring in matrices which arise during intermediate computations. Recall that we write $\|U\|$ to mean the magnitude of the largest entry in an integer matrix $U$.

Lemma 7 Let $U, A$ and $H$ be $n \times n$ nonsingular integral matrices which satisfy $U A=H$ where $H$ is in Hermite normal form. If $d=|\operatorname{det}(A)|$, then

$$
\left\|H^{\mathrm{adj}}\right\| \leq d .
$$

If $B$ is any other $n \times n$ integral matrix, then

$$
\|H B\| \leq d\|B\| \text {. }
$$

Lemma 8 There exists a deterministic algorithm that takes as input a $2 m \times m$ matrix

$$
A=\left[\frac{H_{1}}{H_{2}}\right],
$$

where both $H_{1}$ and $H_{2}$ are $m \times m$ of rank $m$ and in Hermite normal form, and produces as output the Hermite normal form $H$ of $A$ together with a unimodular matrix $U$ such that $U A=H$. If $d$ bounds both $\operatorname{det}\left(H_{1}\right)$ and $\operatorname{det}\left(H_{2}\right)$ then $\|U\| \leq d^{2}$. The cost of the algorithm is $O\left(m^{\theta} \mathrm{B}(\log d)\right)$ bit operations.

Proof Compute the Hermite normal form $\bar{H}$ of

$$
\bar{A}=\left[\begin{array}{l|l}
H_{1} & \\
\hline H_{2} & I_{m}
\end{array}\right] .
$$

By Theorem 5 the cost of this is $O\left(m^{\theta} \mathrm{B}(\log d)\right)$ bit operations. We will compute $U$ as $U \leftarrow \bar{H} \bar{A}^{-1}$. First we establish the bound on $\|U\|$. Let $d_{1}=\operatorname{det}\left(H_{1}\right)$ and $d_{2}=\operatorname{det}\left(H_{2}\right)$, then

$$
\begin{aligned}
& \|U\|=\left\|\vec{H} \bar{A}^{-1}\right\| \\
& \leq d_{1} \| \bar{A}^{\operatorname{adj}_{\left(1 / d_{1}\right)} \|} \\
& \leq\left\|\bar{A}^{\operatorname{adj}}\right\| \\
& =\left\|\left[\begin{array}{c|c}
H_{1}^{\mathrm{adj}} & \\
\hline-H_{2} H_{1}^{\mathrm{adj}} & d_{1} I
\end{array}\right]\right\| \\
& \leq \max \left(\left\|H_{1}^{\mathrm{adj}}\right\|,\left\|H_{2} H_{1}^{\mathrm{adj}}\right\|, d_{1}\right) \\
& \leq \max \left(d_{1}, d_{2} d_{1}, d_{1}\right) \\
& \leq d^{2} \text {. }
\end{aligned}
$$

where lines (6) and (7) follow from Lemma 7. The matrix $U$ can now be computed in $O\left(m^{\theta} \mathrm{B}(m \log m\|A\|)\right)$ bit operations using a standard homomorphic imaging scheme.

\subsection{A Preconditioning Algorithm}

Let $A$ be an $n \times m$ rank $m$ integral input matrix. We can write $A$ using a block decomposition as

$$
\left[\begin{array}{c}
A_{1} \\
A_{2} \\
\vdots \\
A_{l} \\
B
\end{array}\right]
$$

where each $A_{2}$ is $m \times m, l=\lfloor n / m\rfloor$, and $B$ is $t \times m$ with $t=n-l m$. The purpose of this section is to give a fast algorithm for transforming $A$ to an equivalent matrix, but where $A_{\imath}$ is nonsingular for $1 \leq i \leq l$. Our result is the following.

Lemma 9 There exists a deterministic algorithm that takes as input an $n \times m$ rank $m$ integral matrix $A$, and returns as output an $n \times n$ permutation matrix $P$ together with a unimodular matrix

$$
R=\left[\begin{array}{cccccc}
I_{m} & & & & & \\
R_{2} & I_{m} & & & & \\
R_{3} & & I_{m} & & & \\
\vdots & & & \ddots & & \\
R_{l} & & & & I_{m} & \\
O & & & & & I_{t}
\end{array}\right]
$$

where $l=\lfloor n / m\rfloor, t=n-l m$, each $R_{\imath}$ is $m \times m$, and the matrix

$$
R P A=\left[\begin{array}{c}
\bar{A}_{1} \\
\bar{A}_{2} \\
\vdots \\
\bar{A}_{l} \\
B
\end{array}\right]
$$

has each $m \times m$ block $\bar{A}_{i}$ nonsingular for $1 \leq i \leq l$. The matrix $R$ will satisfy $\|R\|=O(m \log m\|A\|)$, and the running time of the algorithm is $O\left(m^{\theta-1} n \mathrm{~B}(m \log m\|A\|)\right)$ bit operations.

Proof Compute a number $z$ such that $b=\prod_{p \leq z}^{p \text { prime }} p>$ $m^{m / 2}\|A\|^{m}$. By Hadamard's bound every minor of $A$ is has magnitude less than $b$. For each prime $p \leq z=$ $O(m \log m\|A\|)$, compute the rank of $(A \bmod p)$, and let $p$ be the first prime for which $A$ has rank $m$ over $\mathbf{Z}_{p}$. This can be accomplished in $O\left(m^{\theta-1} n \mathrm{~B}(b)\right)$ bit operations using an algorithm of Ibarra, Moran and Hui [7, 1982]. The algorithm of Ibarra et al. [7, 1982] also returns a maximal set of linearly independent rows over $\mathbf{Z}_{p}$, and this gives the permutation matrix $P$ such that $P A$ can be written as in (8) with $A_{1}$ nonsingular over $\mathbf{Z}$ and $\mathbf{Z}_{p}$. Fix some $j$ with $2 \leq j \leq l$. Our goal is to compute a matrix $R_{j}$ such that $\bar{A}_{j}=\overline{A_{j}}+R_{j} A_{1}$ is nonsingular. Note that if $(\vec{A}, \bmod p)$ is nonsingular over $\mathbf{Z}_{p}$, then $\bar{A}_{j}$ will be nonsingular over $\mathbf{Z}$. Our approach is to find an $R_{\jmath}$ with entries between 0 and $p-1$ such that $\left(\bar{A}_{j} \bmod p\right)$ is nonsingular over $\mathbf{Z}_{p}$. Since $A_{1}$ is nonsingular over $\mathbf{Z}_{p},\left(\bar{A}_{\jmath} \bmod p\right)$ will be nonsingular over $\mathbf{Z}_{p}$ if and only $\left(\bar{A}_{\jmath} A_{1}^{-1} \bmod p\right)$ is nonsingular over $\mathbf{Z}_{p}$. 
Working $\bmod p$, decompose $\left(A_{3} A_{1}^{-1} \bmod p\right)$ as the sum of a unit upper triangular matrix $U_{3}$ and lower triangular matrix $L_{\jmath}$. Set $R_{\jmath}=\left(-L_{\jmath} \bmod p\right)$. Then entries in $R_{j}$ are between 0 and $p-1$ and

$$
\begin{aligned}
\bar{A}_{j} A_{1}^{-1} & \equiv\left(A_{j} A_{1}^{-1}+R_{\jmath}\right) \bmod p \\
& \left.\equiv\left(\left(U_{j}+L_{\jmath}\right)\right)-L_{\jmath}\right) \bmod p \\
& \equiv U_{\jmath} \bmod p
\end{aligned}
$$

whence $\left(\bar{A}_{3} A_{1}^{-1} \bmod p\right)$ is nonsingular over $\mathbf{Z}_{p}$. The cost of computing $\left(A_{j} A_{1}^{-1} \bmod p\right)$ for $2 \leq j \leq l$ is bounded by $O\left(m^{\theta-1} n \mathrm{~B}(\log p)\right)$ bit operations.

\subsection{A Special Case of the Algorithm}

In this section we develop our algorithm to compute premultiplier matrices for a special class of input matrices. Fix some positive integer parameters $m$ and $d$, and let $\mathcal{T}_{l}=$ $\mathcal{T}_{l}[m, d]$ be the set of all $l m \times m$ rank $m$ integral matrices which can be written using a block decomposition as

$$
\left[\begin{array}{c}
A_{1} \\
A_{2} \\
\vdots \\
A_{l}
\end{array}\right]
$$

where each $m \times m$ block $A_{i}$ is either the zero matrix or is nonsingular and in Hermite normal form, and where $d$ is a positive multiple of $\operatorname{det}\left(A_{2}\right)$ for $1 \leq i \leq l$. Let $H(l)=$ $H[m, d](l)$ denote a function which bounds the number of bit operations required to compute, for a given input matrix $A \in \mathcal{T}_{l}[m, d]$, a unimodular matrix $U$ such that $U A=H$, the Hermite normal form of $A$. Our result is the following.

Theorem $10 H[m, d](l) \ll l \log (2 l) m^{\theta} \mathrm{B}(\log (2 l) \log m d)$.

Our proof of Theorem 10 has two parts. First, we prove the existence of a deterministic algorithm that constructs a candidate for $U$ that will both be sparse and have small entries. For the second part, we analyze the complexity of the algorithm.

We need to define some notation. A $p m \times q m$ matrix $X$ can be written using a block decomposition as

$$
\left[\begin{array}{cccc}
\bar{X}_{11} & \bar{X}_{12} & \cdots & \bar{X}_{1 q} \\
\bar{X}_{21} & \bar{X}_{22} & & \\
\vdots & & \ddots & \\
\bar{X}_{p 1} & & & \bar{X}_{p q}
\end{array}\right]
$$

where each block is $m \times m$. We denote the submatrix $\bar{X}_{2 j}$ by $\operatorname{block}(X, i, j)=\operatorname{block}_{m}(X, i, j)$. For $1 \leq i \leq p$, we also define $\mathrm{L}(X, i)=\mathrm{L}_{m}(X, i)$ by

$\mathrm{L}(X, i):=\{j: 1 \leq j \leq q, \operatorname{block}(X, i, j)$ is not the zero matrix $\}$.

The quantity $\sum_{i=1}^{p}|\mathrm{~L}(X, i)|$ indicates the degree of sparsity of the matrix $X$, that is, the number of nonzero $m \times m$ blocks in the decomposition (10).

Lemma 11 There exists a deterministic algorithm that takes as input an $2^{k} m \times m$ integral matrix $A \in \mathcal{T}_{2^{k}}[m, d]$, and produces as output:

- the Hermite normal form $H$ of $A$,

- a unimodular pre-multiplier $U$ such that $U A=H$,
- the lists $\mathrm{L}(U, i)$ for $1 \leq i \leq 2^{k}$.

If the last $t m$ rows of $A$ are zero, then $U$ can be written as $\operatorname{diag}\left(\bar{U}, I_{t m}\right)$. Furthermore, $\sum_{i=1}^{2^{k}}|\mathrm{~L}(U, i)| \leq 2^{k}(k+1)$, and if $d$ is a positive integer multiple of $\operatorname{det}\left(A_{i}\right)$ for $1 \leq i \leq 2^{k}$, then $\|U\| \leq\left(m d^{2}\right)^{k}$.

Proof We prove the existence of an algorithm which satisfies the requirements of Lemma 11 by induction on $k$. For the initial case $k=0$, set $U \leftarrow I_{m}, H \leftarrow A$ and $L_{1} \leftarrow\{1\}$. The bounds on $\sum_{i=1}^{2^{k}}\left|L_{\imath}\right|$ and $\|U\|$ are trivially satisfied. Assume the lemma holds for $k=N$. To prove the lemma holds for $k=N+1$, let $A$ be a $2^{N+1} m \times m$ input matrix in $\mathcal{T}_{2} N+1$. Write $A$ in block form as

$$
\left[\frac{\hat{A}_{1}}{\hat{A}_{2}}\right]
$$

where $\hat{A}_{1}$ and $\hat{A}_{2}$ are $2^{N} m \times m$ and in $\mathcal{T}_{2^{N}}$. By induction, there exists a deterministic algorithm that computes $2^{N} m \times$ $2^{N} m$ unimodular $U_{1}$ and $U_{2}$ such that

$$
\left[\begin{array}{c|c}
U_{1} & \\
\hline & U_{2}
\end{array}\right]\left[\begin{array}{c}
\hat{A}_{1} \\
\hline \hat{A}_{2}
\end{array}\right]=\left[\begin{array}{c}
H_{1} \\
O \\
\vdots \\
O \\
\hline H_{2} \\
O \\
\vdots \\
O
\end{array}\right]
$$

where $H_{1}$ and $H_{2}$ are $m \times m$ and in Hermite normal for$\mathrm{m}$ with determinants bounded by $d$. We also get the lists $\mathrm{L}\left(U_{1}, i\right)$ and $\mathrm{L}\left(U_{2}, i\right)$ for $1 \leq i \leq 2^{N}$. Compute a $2 m \times 2 m$ unimodular matrix

$$
\left[\begin{array}{l|l}
P & Q \\
\hline R & S
\end{array}\right],
$$

with each block $m \times m$, and that satisfies

$$
\left[\begin{array}{c|c}
P & Q \\
\hline R & S
\end{array}\right]\left[\begin{array}{l}
H_{1} \\
\hline H_{2}
\end{array}\right]=\left[\begin{array}{c}
H \\
\hline O
\end{array}\right]
$$

where $H$ is $m \times m$ and in Hermite normal form.

Remark 1: By Lemma 8, the cost of producing $P, Q, R$ and $S$ is bounded by $O\left(m^{\theta} \mathrm{B}(\log d)\right)$ bit operations.

Embed $P, Q, R$ and $S$ into the $2^{N+1} m \times 2^{N+1} m$ identity matrix and compute $U$ as

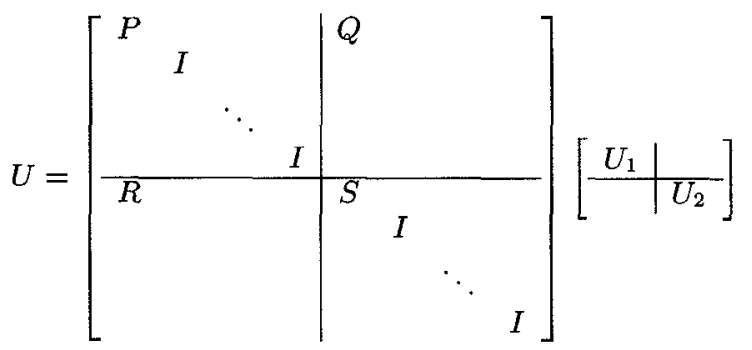

so that $U$ is unimodular and satisfies

$$
U A=\left[\begin{array}{c}
H \\
O \\
\vdots \\
O
\end{array}\right]
$$


By the induction hypothesis, $\left\|U_{1}\right\|,\left\|U_{2}\right\| \leq\left(m d^{2}\right)^{N}$, and by Lemma 7 , entries in $P, Q, R$ and $S$ are bounded by $d^{2}$. It follows that $\|U\| \leq m \cdot d^{2} \cdot\left(m d^{2}\right)^{N}=\left(m d^{2}\right)^{N+1}$ as required.

Remark 2: The matrix multiplication in (12) requires $2^{N}$ multiplications of pairs of $2 m \times 2 m$ blocks. Since $\|U\| \leq\left(m d^{2}\right)^{N+1}$, entries in $U$ are bounded in length by $\log \|U\|=O(N \log m d)$ bits - this bounds as well the length of entries in the matrices $P, Q, R, S$ and $U_{1}, U_{2}$. This leads to a bound of $O\left(2^{N} \mathrm{MM}(m) \mathrm{B}(N \log m d)\right)$ bit operations to accomplish the multiplication in (12).

For convenience, define $b=2^{N}$. By (12), we have $\mathrm{L}(U, i)=$ $\mathrm{L}\left(U_{1}, i\right)$ for $2 \leq i \leq b$. Similarly, for $2 \leq i \leq b$, we can obtain $\mathrm{L}(U, b+i)$ from the list $\mathrm{L}\left(U_{2}, i\right)$ by adding $2^{N}$ to each entry. The lists $L(U, 1)$ and $L(U, b+1)$ can be computed by examining each of the blocks block $(U, 1, j)$ and block $(U, b+$ $1, j)$ for $1 \leq j \leq 2^{N+1}$.

Remark 3: The cost of examining each of the blocks block $(U, 1, j)$ and $\operatorname{block}(U, b+1, j)$ for $1 \leq j \leq 2 b$ is bounded by $O(b m \log d)$ bit operations, and this bounds the total cost of computing $L(U, i)$ for $1 \leq i \leq 2 b$.

It remains to bound the quantity $\sum_{2=1}^{2 b}|\mathrm{~L}(U, i)|$. It follows from the construction of $U$ in (12) that both $|\mathrm{L}(U, 1)|$ and $|\mathrm{L}(U, b+1)|$ are bounded by $\left|\mathrm{L}\left(U_{1}, 1\right)\right|+\left|\mathrm{L}\left(U_{2}, 1\right)\right|$. We get

$$
\begin{aligned}
& \sum_{i=1}^{2 b}|\mathrm{~L}(U, i)| \\
& \quad=|\mathrm{L}(U, 1)|+|\mathrm{L}(U, b+1)|+\sum_{i=2}^{b}(|\mathrm{~L}(U, i)|+|\mathrm{L}(U, b+i)|) \\
& \quad=2\left(\left|\mathrm{~L}\left(U_{1}, 1\right)\right|+\left|\mathrm{L}\left(U_{2}, 1\right)\right|\right)+\sum_{i=2}^{b}\left(\left|\mathrm{~L}\left(U_{1}, i\right)\right|+\left|\mathrm{L}\left(U_{2}, i\right)\right|\right) \\
& \quad \leq \mathrm{L}\left(U_{1}, 1\right)|+| \mathrm{L}\left(U_{2}, 1\right) \mid+\sum_{i=1}^{b}\left(\left|\mathrm{~L}\left(U_{1}, i\right)\right|+\left|\mathrm{L}\left(U_{2}, i\right)\right|\right) \\
& \leq 2^{N}+2^{N}+22^{N}(N+1) \\
& =2^{N+1}+2^{N+1}(N+1) \\
& =2^{N+1}(N+2)
\end{aligned}
$$

as required.

We now return to the proof of Theorem 10 . We claim that for $H(l)=H[m, d](l)$ we have

$$
H(l) \leq 2 H(l / 2)+c l m^{\theta} \mathrm{B}\left(\log _{2}(2 l) \log m d\right)
$$

for some absolute constant $c$. Let $A$ be in $\mathcal{T}_{l}$ with Hermite normal form $H$. By augmenting $A$ with at most $(l-1) m$ rows of zeros, we may assume that $l=2^{N+1}$ for some $N \in \mathbf{Z}$. Rewrite (13) with $l=2^{N+1}$ to get

$$
\left.H\left(2^{N+1}\right) \leq 2 H\left(2^{N}\right)+c 2^{N+1} m^{\theta} \mathrm{B}((N+2)) \log m d\right)
$$

The algorithm presented in Lemma 11 reduces the problem of computing a unimodular $U$ with $U A$ in Hermite normal form to two subproblems in $\mathcal{T}_{2}$ and some combining steps. Thus, to prove (14), it is sufficient to show that the cost of the combining steps is bounded by $O\left(2^{N+1} m^{\theta} \mathrm{B}((N+\right.$
2) $\log m d$ ) bit operations - but this follows from Remarks 1,2 and 3 . Iterate (14) to get

$$
\begin{aligned}
& H\left(2^{N+1}\right) \\
& \quad \leq 2 H\left(2^{N}\right)+c 2^{N+1} m^{\theta} \mathrm{B}((N+2) \log m d) \\
& \leq 4 H\left(2^{N-1}\right)+c m^{\theta} \mathrm{B}((N+2) \log m d)\left(2^{N+1}+2 \cdot 2^{N}\right) \\
& \quad \vdots \\
& \quad \leq 2^{N+1} H(0)+c m^{\theta} \mathrm{B}((N+2) \log m d) \sum_{\imath=0}^{N+2} 2^{\imath} 2^{N+2-\imath} \\
& \quad \ll 2^{N} N m^{\theta} \mathrm{B}(N \log m d)
\end{aligned}
$$

and we have our result since $l=2^{N+1}$.

\subsection{The Premultiplier Algorithm}

Theorem 12 There exists a deterministic algorithm that takes as input an $n \times m$ rank $m$ integral matrix $A$, and produces as output the Hermite normal form $H$ of $A$ together with a unimodular pre-multiplier matrix $U$ that satisfies $U A=H$. The running time of the algorithm is bounded by $O\left(m^{\theta-1} n \log (2 n / m) \mathrm{B}(\log (2 n / m) m \log m\|A\|)\right)$ bit operations.

Proof The algorithm has four steps. First, use the algorithm developed in Subsection 4.1 to compute an $n \times n$ permutation matrix $P$ and an $n \times n$ unimodular matrix

$$
R=\left[\begin{array}{cccccc}
I_{m} & & & & & \\
R_{2} & I_{m} & & & & \\
R_{2} & & I_{m} & & & \\
\vdots & & & \ddots & & \\
R_{l} & & & & I_{m} & \\
O & & & & & I_{t}
\end{array}\right]
$$

such that

$$
\vec{A}=R P A=\left[\begin{array}{c}
\bar{A}_{1} \\
\bar{A}_{2} \\
\vdots \\
\overline{\bar{A}}_{l} \\
B
\end{array}\right]
$$

where each $m \times m$ submatrix $\bar{A}_{2}$ is nonsingular, $l=\lfloor n / m\rfloor$, and $B$ is $t \times m$ where $t=n-l m$. By Lemma 9 , the cost of this will be bounded by $O\left(m^{\theta-1} n \mathrm{~B}(m \log m\|A\|)\right)$ bit operations. Secondly, compute an $(m+t) \times(m+t)$ unimodular matrix $U_{l}$ such that

$$
U_{l}\left[\begin{array}{c|c}
\bar{A}_{l} & O \\
\hline B & I_{t}
\end{array}\right]=\left[\begin{array}{c|c}
H_{l} & * \\
\hline O & *
\end{array}\right]
$$

with the matrix on the right hand side in Hermite normal form. In particular, $H_{l}$ will be $m \times m$ and in Hermite normal form. Thirdly, compute matrices $U_{1}, U_{2}, \ldots, U_{l-1}$, each $m \times$ $m$ and unimodular, and satisfying

$$
\left[\begin{array}{ccccc}
U_{1} & & & & \\
& U_{2} & & & \\
& & \ddots & & \\
& & & U_{l-1} & \\
& & & & U_{l}
\end{array}\right] \bar{A}=\left[\begin{array}{c}
H_{1} \\
H_{2} \\
\vdots \\
H_{l} \\
O
\end{array}\right]
$$


with each $H_{2}$ an $m \times m$ matrix in Hermite normal for$\mathrm{m}$ for $1 \leq i \leq l$. By Theorem 5, the cost of computing $U_{\imath}$ and $H_{i}$ for $1 \leq i \leq l$ is bounded by $O\left(l m^{\theta} \mathrm{B}(m \log m\|\bar{A}\|)\right.$ bit operations. Compute the quantity $d=\operatorname{lcm}\left(\operatorname{det}\left(H_{1}\right), \operatorname{det}\left(H_{2}\right), \ldots, \operatorname{det}\left(H_{l}\right)\right)$. By Hadamard$\mathrm{s}$ inequality on determinants, we will have $\log d=$ $O(m \log m\|\bar{A}\|)$. Fourthly, use the algorithm described in Subsection 4.2 to compute an $l m \times l m$ unimodular $S$ satisfying

$$
S\left[\begin{array}{c}
H_{1} \\
H_{2} \\
\vdots \\
H_{l}
\end{array}\right]=\left[\begin{array}{c}
H \\
O
\end{array}\right]
$$

where

$$
H
$$

is

$m \times m$ and in Hermite normal form. By Theorem (10), this costs $O\left(m^{\theta-1} n \log (2 n / m) \mathrm{B}(\log (2 n / m) m \log m\|\bar{A}\|)\right)$ bit operations, which bounds the total cost up until this point. Finally, set

$$
U=\left[\begin{array}{ll}
S & \\
& I_{t}
\end{array}\right]\left[\begin{array}{ccccc}
U_{1} & & & & \\
& U_{2} & & & \\
& & \ddots & & \\
& & & U_{l-1} & \\
& & & & U_{l}
\end{array}\right] R P
$$

so that $U$ is unimodular with $U A$ the Hermite normal for$\mathrm{m}$ of $A$. It remains to establish a bound on the cost of the matrix multiplications in (15). By Lemma 11, we have $2 \sum_{i=1}^{k}\left|\mathrm{~L}_{m}(S, i)\right| \leq 2((2 n / m) \log (4 n / m))$, which, because of the special structure of $R$ and the second matrix in the product (15), bounds the number of pairs of $m \times m$ matrix blocks which need to be multiplied. By the bounds established on the magnitudes of entries in $S, U_{1}, U_{2}, \ldots, U_{l}$, we have

$$
\log \|U\|=O(\log (2 n / m) m \log m\|\bar{A}\|),
$$

leading to a cost for the multiplications in (15) of $O\left(m^{\theta-1} n \log (2 n / m) \mathrm{B}(\log (2 n / m) m \log m\|\bar{A}\|)\right)$ bit operations. By Lemma 9 , the entries in $R$ will be bounded in magnitude by $\mathrm{cm} \log m\|A\|$ bits for some absolute constant $c$. This leads to the bound $\|\bar{A}\| \leq \mathrm{cm}^{2}\|A\| \log m\|A\|$. The result now follows by noting that $m \log m\|\bar{A}\|=$ $O(m \log m\|A\|)$.

Acknowledgement: Thanks to two anonymous referees for many useful corrections and suggestions.

\section{References}

[1] Aho, A. V., Hopcroft, J. E., and Ullman, J. D. The Design and Analysis of Computer Algorithms. Addison-Wesley, 1974.

[2] Coppersmith, D., and Winograd, S. Matrix multiplication via arithmetic progressions. Journal of Symbolic Computation 9 (1990), 251-280.

[3] Domich, P. D., Kannan, R., And Trotter, JR., L. E. Hermite normal form computation using modulo determinant arithmetic. Mathematics of Operations Research 12, 1 (Feb. 1987), 50-59.

[4] Hafner, J. L., ANd McCurley, K. S. Asymptotically fast triangularization of matrices over rings. SIAM Journal of Computing 20, 6 (Dec. 1991), 1068-1083.
[5] Hermite, C. Sur l'introduction des variables continues dans la théorie des nombres. J. Reine Angew. Math. 41 (1851), 191-216.

[6] Hu, T. C. Integer Programming and Network Flows. Addison-Wesley, Reading, MA, 1969.

[7] Ibarra, O., Moran, S., AND Hui, R. A generalization of the fast LUP matrix decomposition algorithm and applications. Journal of Algorithms 3 (1982), 45-56.

[8] Iliopoulos, C. S. Worst-case complexity bounds on algorithms for computing the canonical structure of infinite abelian groups and solving systems of linear diophantine equations. SIAM Journal of Computing 18, 4 (Aug. 1989), 670-678.

[9] Iliopoulos, C. S. Worst-case complexity bounds on algorithms for computing the canonical structure of $\mathrm{fi}$ nite abelian groups and the Hermite and Smith normal forms of an integer matrix. SIAM Journal of Computing 18, 4 (Aug. 1989), 658-669.

[10] Newman, M. Integral Matrices. Academic Press, 1972.

[11] Schönhage, A., ANd Strassen, V. Schnelle Multiplikation grosser Zahlen. Computing (1971), 281-292.

[12] Storjohann, A., and Labahn, G. Preconditioning of rectangular polynomial matrices for efficient Hermite normal form computation. In Proceedings of ISSAC'95 (Montreal, Canada, 1995), pp. 119-125.

Arne StorJohann is an Assistant in the Institute for Scientific Computation at ETH-Zurich. He holds B.Math. and M.Math. degrees from the University of Waterloo, Canada. His research interests are in Symbolic Computation, Linear Algebra, Analysis of Algorithms, and Matrix Normal Forms.

George Labahn is an Associate Professor in the Department of Computer Science at the University of Waterloo. Prof. Labahn holds B.Sc. (Math), M.Sc.(CS), Ph.D (CS) degrees from the University of Alberta. His research interests are in Symbolic computation, Linear systems theory, Rational Approximants, and Matrix Normal Forms. 